\title{
INFLUENCIAS POLÍTICAS Y EMPRESARIALES EN LA PRENSA ESPAÑOLA. EL CAMBIO DE DIRECCIÓN EN EL PAÍS
}

Marián Alonso González: Universidad de Sevilla. España. malonsog@us.es http://orcid.org/0000-0003-2676-0449

\section{RESUMEN:}

Este artículo se enfoca en torno a una revisión hemerográfica de la prensa nacional e internacional con objeto de exponer las causas atribuidas al cambio de director en el diario El País. Un acusado descenso en el número de lectores y de inversión en publicidad, así como influencias políticas son las ideas más repetidas por los distintos periódicos, de forma que del análisis realizado se concluye que el modelo mercantilista sigue funcionando en los medios impresos y que la prensa ya no es el cuarto poder, sino parte del primero al relacionarse directamente con cuestiones económicas y políticas.

PALABRAS CLAVE: El País - Política - Economía - Lectores - Digital - Periódicos Online

\section{POLITICS AND BUSINESS INFLUENCES IN THE SPANISH PRESS. CHANGE OF DIRECTOR IN THE NEWSPAPER 'EL PAIS'}

\section{ABSTRACT:}

This paper focuses on a review about the national and international news about the reasons to Antonio Caño's appointment as new Director of El País, the newspaper with more readers in Spain. Economic and political influences are the ideas that more repeat themselves in the digital national and international press. In practice therefore, the model mercantilist continues working in the printed media, the press already is not the fourth power, the press is a part of the first one since it relates to economic and political questions.

KEY WORDS: El País - Politics - Economics - Readers - Digital - Newspapers Online 


\section{INTRODUCCIÓN}

Los medios de comunicación son entidades con un gran peso de la esfera pública, lo que se traduce en una gran relevancia social. Periodismo y Política van de la mano, y en esta relación singular se impone el modelo mercantilista que determina la estructura empresarial a la que pertenece el medio de comunicación, de forma que "la situación financiera de la empresa marcará la pauta de los trabajadores, y el periodista no se podrá quedar al margen" (Labio, 2001, p.87).

La prensa ya no es el cuarto poder, sino que se ha convertido en parte del primero al relacionarse directamente con cuestiones económicas y políticas, señala Labio, y en este sentido, la independencia del profesional de la información se sustituye por una clara adhesión a la ideología del medio para el que trabaja.

Esta ausencia de independencia provoca que el tratamiento informativo del medio se halle condicionado estructuralmente por el contexto sociopolítico, pero también por el condicionante económico que impone la dictadura de la publicidad. Este fenómeno no es nuevo, de hecho, en España podemos constatar la existencia de medios nacionales que siguen el bipartidismo impuesto por PP y PSOE, nos referimos, a los diarios El Mundo y El País.

La caída en picado de las ventas de ejemplares y de los ingresos por publicidad, así como la presión ejercida por el poder gobernante, ha provocado cambios en la estructura de dirección de los periódicos españoles, cobrándose la destitución en el plazo de un mes de los directores de El Mundo, El País y La Vanguardia. Cambios que abren un periodo convulso y de inestabilidad en las redacciones que algunos analistas vinculan con el auge de la prensa digital y el rescate directo a las editoras protagonizado por parte del poder político.

A lo largo de este artículo, y haciendo uso de una metodología basada en una revisión bibliográfica y hemerográfica, vamos a intentar apuntar los factores que han incidido en el cambio de dirección de El País, por ser el diario de tirada nacional con un mayor índice de lectores. Según el Estudio General de Medios correspondiente al primer trimestre de 2015, entre los medios generalistas El País mantiene el liderazgo como diario más leído (1.533.000 lectores diarios) "aunque continúa con un desplome de sus audiencias, que en esta ocasión supera el 13\% respecto a la misma ola del EGM'14, siendo la caída más acusada dentro del medio" (Ymedia, 2015).

Centramos este artículo, pues, en recoger lo publicado sobre el cambio de director en la prensa nacional e internacional, a fin de poder dibujar un completo perfil sobre las causas que motivaron el cese de Javier Moreno, el nombramiento del actual director, Antonio Caño, y las implicaciones que esta operación interna del medio ha tenido a nivel político y económico.

\subsection{Prensa, poder político y económico.}

La estructura de la información es la materia que tiene por objeto "el estudio del 
sistema de medios de comunicación social, tanto en su organización y funcionamiento interno, como en sus interacciones con otros subsistemas del sistema social' (Quirós, 1991, p.307).

En base a esta definición, acota Ramón Reig (2009, p.388) que es gracias a la estructura de la información que los profesionales pueden conocer "el mundo y su mundo, es decir, los entresijos de su profesión y el entorno en el que esos entresijos están inmersos". De forma que es en esta interrelación entre información, política y economía donde se evidencia la interrelación de un sistema más complejo.

Esta interrelación entre elementos informativos, económicos y políticos evidencia que el concepto de estructura no está aislado y que hay que enmarcarlo dentro del sistema de Economía de Mercado, el cual, según afirma Marciano, está fundamentado por la diversificación de medios de comunicación y se encuentra en condiciones óptimas para:

Afrontar y esclarecer algunos de estos cambios capitales de nuestra época, pues su objeto es el de articular economía, política, comunicación y cultura dentro del mismo proceso de análisis, cuyo principal foco de atención debe ser la forma general y las características que adquiere el capitalismo mundial como sistema global (Marciano, 1992, p.20).

En este mismo sentido manifiesta Labio (2006) que la presión económica puede venir marcada desde el momento que el medio pertenece a una estructura empresarial con unos intereses encaminados a obtener un beneficio económico, de manera que la publicidad y la situación financiera de la empresa marcarán la pauta de los trabajadores.

A nivel político ocurre una situación semejante y, en este sentido, "vemos que los medios de comunicación defienden los intereses de un partido o de otro" (Labio, 2006, p. 87), ello es debido a que aquellos que poseen el poder tienen más opciones de "influir en la estructura y funcionamiento de la sociedad" (Cachero, 2006, p.85).

En esta misma línea, afirma Fernando Quirós que existe:

Una conexión indudable entre los centros de poder del sistema internacional y los verdaderos emisores del proceso de la comunicación internacional. La identificación del discurso informativo con el poder viene tanto de los gobiernos como de las empresas privadas (Quirós, 1991, p.74).

Poder, control y clase dominante son conceptos interrelacionados entre sí, de forma que Manuel Castells manifiesta que:

El poder no se localiza en una esfera o institución social concreta, sino que está repartido en todo el ámbito de la acción humana. Sin embargo, hay manifestaciones concentradas de relaciones de poder en ciertas formas sociales que condicionan y enmarcan la práctica del poder en la sociedad en general imponiendo la dominación. El poder es relacional, la dominación es institucional (Castells, 2009, p.142). 
El uso de la prensa para conseguir unos determinados objetivos afines a los intereses de la clase dominante no es un fenómeno nuevo en la historia de la Comunicación, de hecho, es el siglo XIX cuando de la mano de la burguesía capitalista se pasa de una etapa en la que predomina la información ideológica a una información mercantil, concibiéndose los medios como una empresa preocupada por la obtención de beneficios económicos.

Sin embargo, es a partir de la II Guerra Mundial cuando asistimos a un proceso de globalización en el ámbito de los medios de comunicación, los cuales, según Manuel Delgado (1998), intentan dominar toda la cadena del proceso hasta llegar a la difusión y relación con los usuarios de la comunicación.

La función esencial de los medios es mantener informada a la opinión pública, pero esta tarea entra en conflicto con los intereses políticos de los gobiernos, es por ello que se restringe y moldea la información que se proporciona a los ciudadanos.

La información periodística juega un papel clave en la estructuración de la percepción ciudadana y en la formación de la opinión pública. En las sociedades avanzadas actuales reafirma su condición de recurso clave para ejercer el poder. Por lo tanto, su control se convierte en un aspecto fundamental para desplegar influencia social (Casero, 2009, p.354).

Esa estructuración de la percepción ciudadana es vista por Lorenzo Gomis (1991) como un método de interpretación de la realidad social, de forma que pone en jaque el valor subjetivo del Periodismo al dar por supuesto que el periodista es parte integrante de una institución comercial que construye noticias.

En esta misma línea se pronuncia Mar de Foncuberta (1995) al aportar un enfoque estructuralista que determina que el principal interés de la empresa periodística es siempre económico y que coincide con la definición que de la industria informativa realiza Herbert Schiller:

Los medios de comunicación social son los brazos ejecutores del sistema. Los gobernantes son capaces de confiar en la ayuda de los medios de comunicación. De cuando en cuando sucede algo y arman un revuelo sobre esto y lo otro, pero si los medios practican algo vagamente parecido a la responsabilidad social, se producirá alarma, cólera y una represalia inmediata en los centros neurálgicos del sistema. Pero como los medios son una parte del sistema de poder, lo controladores no tienen nada de qué preocuparse Mosco (1983, p.134).

En resumen, el tratamiento de la información está supeditado a las estructuras políticas y empresariales, algo que implica un fuerte ataque a la autonomía y libertad del periodista y que implica que sectores ajenos a la comunicación participen en la construcción de los textos informativos.

\section{OBJETIVO}

El objetivo de la presente investigación es poner de manifiesto cómo las influencias 
políticas y empresariales, tal y como ya había ocurrido en otros diarios como El Mundo y La Vanguardia, propician el cambio de director en El País, el periódico con mayor índice de lectores de España.

\section{METODOLOGÍA}

Desde los enfoques que nos proporciona la revisión hemerográfica del caso se realizarán valoraciones fundamentadas en el análisis del discurso para desarrollar los planteamientos reflexivos entorno al debate. Así pues, el método de trabajo elegido para la aproximación al problema de investigación utiliza técnicas de carácter cualitativo, amén de una revisión de la literatura sobre las relaciones existentes entre prensa y poder político y económico.

La aproximación al objeto de estudio se hace desde diferentes frentes que permiten una investigación más ajustada a la realidad comunicativa. En primer lugar, se ha llevado a cabo una revisión bibliográfica para enmarcar el tema dentro de un contexto teórico. A continuación, hemos realizado una exhaustiva labor de documentación y rastreo de noticias relacionadas con el relevo direccional en El País en un total de 220 periódicos, de los que 37 eran nacionales y 183 internacionales. Para la prensa internacional, nos hemos centrado en las principales potencias europeas (Francia, Italia, Alemania e Inglaterra), Estados Unidos y Latinoamérica, países a los que da cobertura la edición internacional del periódico.

A través de un muestreo estratégico se han analizado las noticias que han aparecido en los medios de comunicación de España (impresos y digitales) tanto en sus secciones de carácter informativo como de opinión (editoriales, blogs, tribunas o columnas) durante el primer cuatrimestre de 2014, meses en los que en los que surgieron los primeros rumores, confirmaciones y repercusiones en torno al cambio de director, a fin de intentar reconstruir cómo y en qué circunstancias se produce este cambio al frente del primer rotativo en España en términos de audiencia.

\section{DISCUSIÓN}

\subsection{El País. Contexto económico.}

Fundado en 1976 por José Ortega Spottorno, Jesús de Polanco y Juan Luis Cebrián, El País pertenece al Grupo Prisa, también propietario de los diarios Cinco Días y $A S$, la cadena radiofónica $S E R$, Digital + y las editoriales Santillana, Alfaguara y Aguilar, entre otros.

Diseñado por Reinhard Gade y Julio Alonso, El País se convirtió en el periódico de la España democrática en unos momentos en los que el tránsito del franquismo a la democracia estaba todavía en pleno desarrollo. A ello contribuyó, decisivamente, el papel jugado durante el intento de golpe de estado del $23-\mathrm{F}$, ya que en plena incertidumbre fue el primer diario en sacar a la calle una edición especial del periódico titulado "El País, con la Constitución", posicionándose claramente y llamando a los ciudadanos a manifestarse en favor de la democracia. 
Durante la década de los 80 , el diario se consolida como líder de la prensa española frente a $A B C$, de tendencia conservadora. A su prestigio contribuyó un riguroso tratamiento de las normas periodísticas y el ser el primero en establecer parámetros internos de control de calidad. Así, fue el primer diario español en crear la figura del "Defensor del lector" y en redactar y publicar un Libro de estilo que se convirtió en un referente dentro del mundo del Periodismo. También estableció varios acuerdos de colaboración con otros periódicos europeos de línea socialdemócrata.

Durante los años 90 tuvo que hacer frente a una nueva situación política y periodística. La tensión propiciada por los escándalos de corrupción del gobierno socialista de Felipe González polarizó tanto a la política española como a la prensa en medios de izquierda y de derecha, encuadrándose a El País dentro del primer grupo. A pesar de ser acusado de defender los intereses socialistas logró mantener su liderazgo como diario generalista más vendido de España, aunque a menor distancia de El Mundo.

Durante los gobiernos de José Luis Rodríguez Zapatero el periódico publicó cada vez más artículos críticos u opuestos a las políticas del gobierno socialista, una tendencia que ha continuado durante el gobierno de Mariano Rajoy y que algunos periodistas, como Sáenz de Ugarte (2014), apuntan que "se ha ido produciendo en paralelo a los crecientes problemas económicos de Prisa, obligada a negociar en posición vulnerable con los grandes bancos del país para renegociar una deuda inmensa".

En el año 2011 el Grupo Prisa registró las mayores pérdidas de su historia, 451 millones de euros, una cifra seis veces mayor a la anotada en 2010. La crisis económica, unida a un cambio en el modelo de consumo informativo, ha provocado la caída del número de lectores, de forma que en España los periódicos acumulan 11.550.000 lectores por jornada, un 2,2\% menos que a finales de 2014. Estas cifras dibujan un escenario marcado por la continua caída en los índices de audiencia, lo que provoca que España sea unos de los países de la Unión Europea con la tasa de lectura más baja de toda Europa, tan sólo superado por Italia, Portugal y Grecia (Infoeuropa, 2014).

Los efectos de la crisis también se han dejado sentir en un descenso de la inversión publicitaria, que en el año 2013 fue del 3,7\% respecto a 2012, un retroceso que es mayor en los medios convencionales, que registran un $8 \%$ que sumado al $15,8 \%$ de caída experimentado en el año anterior hace que el descenso de ingresos en los periódicos haya sido de casi un $25 \%$ en tan sólo dos años, según datos del Estudio Infoadex de la Inversión Publicitaria en España 2014 (Prnoticias, 2014).

A los malos resultados económicos se suma también en los últimos años una parálisis del sistema de subvención central, la oclusión bancaria y una disfución editorial, lo que provoca que el rescate de las grandes empresas editoras (Prisa, Unidad Editorial, Grupo Godó y Vocento, entre otras) haya dependido del poder político, y esta clara dependencia política se ha visto reflejada en cambios en la estructura de dirección de las cabeceras. 
Al igual que ocurrió con la destitución de Pedro J. Ramírez al frente del diario El Mundo, y a tan sólo tres semanas del primero, el cambio de dirección en El País ha suscita numerosas elucubraciones a nivel nacional e internacional. La principal de ellas se centra en la figura de Nicolás Berggruen, propietario de Berggruen Holdings y consejero delegado de Liberty Acquisitions Honding Corp, el fondo de inversión americano que en 2010 se hacía con el paquete mayoritario del Grupo Prisa.

Lo que en principio pasa por una simple operación inversora finaliza en la venta del $57,7 \%$ del capital de la nueva Prisa y su conversión en acciones con todos los derechos sociales, incluido el derecho al voto. Mediante esta operación Berggruen y su socio en Liberty, Martin Franklin, se convierten en los miembros del Comité de Dirección que controlan el mayor porcentaje de acciones -el siguiente es Juan Luis Cebrián con un $0,28 \%-$, cerca de un $4 \%$ cada uno, así como el mayor índice de stock options ${ }^{1}$ de la compañía, más de un 7,5\% por cabeza.

La inversión, que se anuncia como "meramente financiera", y de la que se llega a decir que los nuevos propietarios no intervendrían en el día a día, provoca cambios en la línea editorial del periódico, al menos en los temas más sensibles "para el nuevo hombre fuerte".

Las informaciones y los editoriales del periódico de Prisa sobre Israel no tienen la radicalidad de antaño, y además hace tiempo que no es posible leer los libelos en forma de artículo o las viñetas furiosamente antisemitas bastante habituales no hace tanto (Jordá, 2014).

Para Jordá, la entrada de Liberty en el accionariado de Prisa explicaría tanto la llegada a Jerusalén como corresponsal del articulista Enric González como el despido del viñetista Romeu, justo un mes antes del anuncio de acuerdo con Liberty, pero después de que suscitase las críticas de varios senadores de Estados Unidos.

Pese a la inyección económica que supone para Prisa la entrada en su accionariado de Liberty, la desesperada situación que atraviesa el conglomerado de medios es una constante informativa durante los últimos años.

En 2008 el grupo mantuvo un saldo positivo en su resultado neto gracias a los 300 millones conseguidos tras vender sus sedes de Gran Vía (Cadena Ser), Miguel Yuste (El País) y Caspe (Radio Barcelona). Sin embargo, debido a las deudas, 2009 se saldó con la venta del $21 \%$ de la plataforma de televisión para abonados Digital Plus a Telefónica por 470 millones y del $22 \%$ a Telecinco por 517 millones. La cadena de Paolo Vasile se hizo además con Cuatro, por 550 millones más (20 Minutos, 2012).

La profunda crisis económica que atraviesa el grupo le lleva a ir despojándose de sus propiedades mediáticas y se traduce en una alta conflictividad laboral que se inicia

\footnotetext{
${ }^{1}$ Una stock option es un derecho que concede la empresa a sus colaboradores a comprar un cierto número de acciones de la misma a un determinado precio (precio de ejercicio) y durante un período de tiempo.
} 
en 2008 cuando el diario aborda una profunda reestructuración que supone su división en tres empresas: contenidos, impresión y servicios, así como la externalización de su departamento comercial.

Tras este hito, en 2010 Prisa externaliza el trabajo de los 300 trabajadores de su departamento de Informática, los cuales dejan de estar en la plantilla de la compañía para pasar a estar en nómina de la empresa Indra. La situación se agrava con el anuncio de un ERE, en octubre de 2012, que supone el despido de un total de 128 trabajadores, 21 prejubilaciones y una disminución del $15 \%$ del sueldo para el resto de una plantilla que queda reducida a 464 trabajadores.

Y todo ello, mientras que el consejero delegado, Juan Luis Cebrián, "se embolsa en torno a 13 millones de euros en 2011, una cantidad que viene a ser todo lo que ingresa el diario por publicidad en dos meses o lo que costaría contratar a 400 nuevos redactores", según publica Pascual Serrano (2009) en Le Monde Diplomatique.

Desde la entrada de Liberty en el accionariado, el plan de reestructuración y eficiencia operativa de Prisa ha abarcado EREs en la Cadena Ser (258 despidos), Prisa TV (784), Cinco Días (21), Prisa Revistas (45) y Prisa Brand Solutions (24). Si a todo ello le sumamos las salidas realizadas en El País, en total los despidos realizados por Prisa alcanzarían los 1.281 de los 2.500 trabajadores que tiene previsto acometer en todo el mundo.

\subsection{Cambio de director}

De todos los conglomerados que conforman el grupo, el diario El País es el que más ha sufrido los despidos, es por ello, que tras el último ERE, la asamblea de trabajadores solicitó la dimisión de su director, Javier Moreno, ya que consideraban que "ha quedado inhabilitado para ejercer el cargo que ostenta y le exige que presente su dimisión inmediatamente por las presiones y amenazas ejercidas contra los trabajadores" (20 Minutos, 2012).

Pocos imaginaron que esa sustitución vendría marcada por la polémica tan solo un año después. A mediados de febrero de 2014 se produce el cese de Moreno y el nombramiento de Antonio Caño (por aquellos entonces corresponsal en Washington) como nuevo director. El nombramiento se realiza en medio de una recusación por parte de casi el $35 \%$ de los trabajadores y precedido por un informe interno en el que exponía por escrito su visión sobre el periódico y sobre los cambios más convenientes a realizar a fin de frenar su galopante pérdida de audiencia e influencia.

Un supuesto "error informático" hace llegar una copia del informe a una cincuentena de periodistas y colaboradores de las distintas delegaciones americanas poniendo al descubierto la sugerencia de Caño de cambiar todo el equipo directivo ya que "no conecta ni con la redacción ni con la sociedad a la que debe abrirse el diario" (Infolibre, 2014). 
El informe propone explícitamente sustituir a Javier Moreno por un "profesional (se supone que de la casa) con trayectoria reconocida y respetado por la Redacción" (Infolibre, 2014) y plantea un esquema con tres directores: uno para el El País papel, otro para la edición digital y un tercero para la edición América. Añade, además, la conveniencia de incorporar jóvenes de 30 años al equipo directivo y critica las "formas y los lujos que aún derrochan miembros del llamado internamente grupo de los coroneles" (Soteras, 2014).

Este informe es interpretado por muchos como "un programa electoral de derechas". Tal nombramiento encajaría en el giro que desde hace tiempo se percibe en la línea editorial de El País, cada día más cercana a las posiciones del Gobierno, especialmente en materia de política económica. Los intereses de Mariano Rajoy son coincidentes con los de grandes empresas y bancos acreedores del gigantesco endeudamiento del periódico (Soteras, 2014).

El nombramiento oficial de Antonio Caño se produce el 26 de febrero mediante un comunicado oficial, aunque no se hace hecho efectivo hasta el 4 de mayo, con motivo del 38 aniversario del periódico.

La llegada de Caño a la dirección de El País provoca numerosos cambios dentro de la plantilla del rotativo:

El nuevo equipo directivo está formado por Eva Sáiz, como subdirectora de la edición impresa; Bernardo Marión, subdirector de la edición digital y Luis Prados como responsable de la edición América. A todos ellos hay que sumar a David Alandete, director adjunto, y que aquellos que integraban el núcleo duro del anterior director fueran destinados a corresponsalías (Lardiés, 2014a).

De esta forma, Vicente Jiménez que era director adjunto fue destinado a Nueva York en sustitución de Sandro Pozzi; Carlos Yárnoz, antiguo subdirector, a París; Juan Manuel Romero, que era quien llevaba el peso de la redacción se queda como corresponsal judicial; Berna G. Harbour, como responsable de Babelia, y Jan Martínez Ahrens se marcha a México donde ya hay un nutrido equipo en el que figuran Luis Prados, Bernardo Marín y Salvador Camarena. México es una las plazas claves del rotativo español, ya que desde ese país se realiza la edición digital de El País durante las noches.

Los cambios realizados por Caño sublevan a parte de la plantilla.

Dos tercios de la redacción de El País, es decir, el $72 \%$, se ha posicionado en contra de la llegada de Eva Saiz como subdirectora, lo que evidencia las tensiones internas que se viven en la sede de Miguel Yuste desde hace dos meses (Vozpópuli, 2014)

Las quejas más comunes, según El Confidencial Digital(2014), apuntan a que nadie en la plantilla la conoce, al tiempo que algunos en la redacción afirman que "sólo ha hecho un máster, ha sido becaria y ha tenido un contrato de colaboradord". 
Sin embargo, el frontal rechazo hacia Saiz viene motivado por unas declaraciones que realiza en relación al pleno celebrado en el Senado después de la publicación de los papeles de Bárcenas, donde la periodista afirmaba que:

Rajoy es un gran parlamentario. No le gusta hablar ante la prensa, pero en el Parlamento la verdad es que se crece y lo hace muy bien. Rajoy venía avisado, sabía que le iban a pedir la dimisión, y él decidió atacar (EI Confidencial Digital, 2014).

Estas declaraciones son interpretadas como una defensa al Presidente del Gobierno y algunos ven en ellas un vaticinio de que la línea del periódico girará a la derecha, más aún si cabe desde el relevo de Javier Moreno.

La frase "Rajoy es un gran parlamentario" demuestra que la nueva subdirectora no tiene un conocimiento del parlamentarismo español acorde a su cargo, se quejan en la redacción. Afirman que se están preparando para una "derechización" de las informaciones durante la etapa de Antonio Caño (Ecoteuve, 2014).

\section{3. Repercusiones en la prensa nacional}

Si bien el nombramiento de Antonio Caño apenas ha tenido repercusión en los medios nacionales impresos, los cuales se han limitado a publicar una breve reseña al respecto, en la prensa digital el cambio en la dirección de El País ha supuesto un auténtico revulsivo y son muchos los medios que ven en la destitución de Javier Moreno un giro a la derecha del mítico rotativo defensor de los ideales de izquierda. Un cambio que se ha ido produciendo en paralelo a los crecientes problemas económicos de Prisa, obligada a negociar en posición vulnerable con los grandes bancos del país para poder renegociar su inmensa deuda.

En este sentido, Libertad Digital (2014) señala que los cambios aparecen explicados de manera pormenorizada en el informe el cual Antonio Caño pide expresamente la sustitución del actual jefe de Nacional, Javier Casqueiro (que sería enviado como corresponsal al Magreb), por José Manuel Romero, que ya ocupó este puesto en el pasado y que es "conocido por sus excelentes relaciones con el ministro Gallardón". $\mathrm{E}$ insiste en que son los propios mandos del diario quienes definen el escrito "un programa electoral de derechas", ya que:

El todavía jefe de El País en América no destaca por tener una política de izquierdas, lo que es visto por la plantilla como un acercamiento de Cebrián al Gobierno de Mariano Rajoy, que en todo momento ha auxiliado a Prisa en su endeudamiento (Libertad Digital, 2014).

Periodistas como Íñigo Sáenz de Ugarte (2014) apuntan que "quizás sea una coincidencia, pero la imagen con la que El País resume la primera jornada del Debate del Estado de la Nación es la foto de un Rajoy haciendo el signo de la victoria". Y señala:

El diario Público arrebató entre 2007 y 2012 un cierto número de lectores al periódico de Prisa, precisamente por la percepción extendida de que este 
había moderado sus puntos de vista. Eso se ha acentuado en los dos últimos años cuando sus editoriales han elogiado algunas de las reformas económicas del Gobierno de Rajoy (Sáenz, 2014).

El informe filtrado de Caño pone de manifiesto que:

El País ha sido arrinconado en un lado del espectro ideológico y esta situación debe ser reconducida desde un giro ideológico a la derecha, alejándose, pues de la posición socialdemócrata adoptada por el periódico desde la década de los 80 en la que decide convertirse en un medio de comunicación cercano al PSOE y sus líderes, desde González a Rubalcaba, pasando por Almunia y Zapatero (Sáenz, 2014).

Esta misma percepción de que se está fraguando un giro a la derecha puede leerse en otros medios digitales. Así, Vozpópulix recoge que con la sustitución de Javier Moreno:

El rotativo da un paso más en un cambio de rumbo, cada vez más criticado por amplias capas de lectores debido a su línea editorial amable con el Gobierno del PP. La evidente rebaja de las críticas a Mariano Rajoy está relacionada, sin duda, con las magníficas relaciones que mantiene Cebrián con la vicepresidenta del Gobierno, Soraya Sáenz de Santamaría (Lardiés, 2014b).

Otros medios online son más agresivos, y así la bitácora de opinión e información sobre medios de comunicación, La mosca mediática (2013), publica que desde que "Rajoy salvó El País sus cambios son evidentes". En relación al Caso Bárcenas, esta bitácora añade que El País.

No machaca al PP, sino al PP de su odiado Aznar, nunca al de Rajoy. Rajoy con esta estrategia se venga de Aznar, al que no le perdona que con su núcleo duro -con Esperanza a la cabeza- le pusieran muy difícil las cosas en su papel de oposición (La mosca mediática, 2013).

De igual forma, señala:

Con este pacto antinatura entre El País y Rajoy, este último consigue que el periódico más leído e influyente no agite la calle, no apoye la revitalización del PSOE o a cualquier nuevo partido político o movimiento social [...] con ello el gallego logra que El País no le meta el dedo en el ojo con sus errores en materia fiscal (La mosca mediática, 2013).

En esta misma línea se pronuncia Daniel Toledo (2014) en Elconfindencial.com donde apunta que Prisa, y por extensión El País, tienen "mucho que agradecer a las grandes empresas españolas que, difícilmente, se atreverían a contrariar al Ejecutivo", y se remonta a junio de 2012 para recordar que Telefónica, Santander y La Caixa "daban un paso al frente para entrar en el capital de Prisa en una suerte de operación rescate de las grandes multinacionales patrias para una compañía acuciada por una deuda de 3.500 millones".

Por su parte, Diario Crítico va más allá y publica un artículo titulado "Cebrián 'pliega' El País a Rajoy para salvar al Grupo Prisa", en cuyo interior podemos leer: 
Cebrián ya sabe cómo 'salvar' al Grupo Prisa del concurso de acreedores: dar al Gobierno de Rajoy en bandeja de plata la cabeza de Javier Moreno y sustituirle en la dirección de El País. Se habla de Antonio Caño, el corresponsal en Washington. Pero, en todo caso, lo de Prisa no es más que una brisa en una tempestad mediática, porque se ha disparado la rumorología sobre un completo tsunami en todos los medios (Diario Crítico, 2014).

Junto a los ríos de tinta publicados sobre el evidente giro ideológico experimentado por el diario, no son pocos los medios nacionales que comienzan a interrelacionar la sustitución de directores que ha experimentado la prensa en cuestión de semanas: La Vanguardia, El Mundo y El País, los diarios con más lectores en España.

Así, el periodista José Sanclemente (2014), en su artículo "¿Quién ha acabado con tres directores de periódico?", analiza los factores comunes de lo que él denomina "un récord de mortandad que da que pensar si no nos estaremos enfrentando a una epidemia". Según Sanclemente, el elemento común en los tres rotativos es que habían caído en picado en ventas y en publicidad, una premisa que si se extrapolara al conjunto de la prensa conllevaría que "todos los directores de este país deberían dejar su puesto al frente de su periódico, pues los lectores y anunciantes están abandonando el papel a marchas forzadas".

Por este motivo, apunta que:

El virus letal debe tener que ver con la orientación de contenidos, una enfermedad de difícil curación [...] sus síntomas son claros: presión política alta, obstrucción del riego publicitario, disfunción editorial y, finalmente, coma cerebral inducido para no provocar lesiones más graves en el aparato informativo (Sanclemente, 2014).

Si bien el cese de José Antich al frente de La Vanguardia fue debido a una imposición del Rey al Grupo Godó, como apunta Periodista Digital (2014); el de Pedro J. Ramírez al frente de El Mundo viene motivada por las presiones de Rajoy y de RCS Mediagroup. El propio periodista apunta en su última carta, nunca publicada en El Mundo, que los motivos de su cese son debidos a "las revelaciones del 'Caso Bárcenas', la financiación ilegal del PP, los SMS cruzados entre Rajoy y su extesorero, y las percepciones recibidas por el marido de Ma Dolores de Cospedal tras la fusión de Caja Castilla-La Mancha" (Vozpópuli, 2014).

Para Pablo Sebastián en su columna de opinión de La República.com, el cese de Javier Moreno al frente de El País se parece al de Pedro J. en El Mundo:

Por cuanto entran en juego poderes políticos, financieros y mediáticos, y porque se pretende un mayor control del total de la información en beneficio de los palacios de Moncloa y Zarzuela -zarandeados por ambos diarios en los últimos meses- que claman por 'la razón de Estado' institucional. Sin embargo, los argumentos oficiales que se ofrecen son otros: los directores de los diarios de papel cesan por la caída de las ventas y de los ingresos publicitarios de sus periódicos y por el obligado giro en favor de sus ediciones en Internet (Periodista digital, 2014b). 
El columnista incluso apunta a la curiosa coincidencia de los ceses de ambos tras los viajes que realiza Rajoy a Washington y a Roma.

Mucho más crítico se muestra Manuel Ortega (2014) en su blog La Tramoya donde, bajo el título de "El País, cautivo y desarmado por Cebrián, se rinde a La Moncloa", publica que los lectores del diario se han debido quedar de piedra tras ver como "el considerado órgano oficioso de la centroizquierda progresista española ha publicado una tribuna firmada por Mariano Rajoy ("El otro lado del Estrecho") en la que el Presidente del Gobierno desglosa varios asuntos de la política africana".

Con su artículo, "Rajoy pone un fin simbólico a la guerra mantenida durante años por el Partido Popular y el Grupo Prisd" y quedan atrás los tiempos en los que "Rajoy ordenaba el boicot a los medios del Grupo Prisa tras las acusaciones de un Jesús Polanco en fase terminal' (Ortega, 2014).

Respecto al cambio ideológico y redaccional, el periodista apunta:

Las perspectivas han generado un estado de tensión permanente en la redacción de El País donde se asiste con asombro a un cambio en la línea editorial que choca con la interiorizada durante años por buena parte de sus miembros [...] Caño ha decidido rodearse de una joven guardia sin experiencia en el día a día de la redacción, pero también sin pasado a fin de poder mantener una línea de apoyo al Ejecutivo pero crítica en aspectos sociales como el aborto o con determinados miembros del gabinete para conservar algunas señas de identidad del periódico hacia sus lectores (Ortega, 2014).

También bastante crítica se muestra Maruja Torres. La que fuera columnista de E/ País, recoge en la sección que protagoniza en la revista Mongolia que "de El País quedan restos, ruinas, excepciones y la fidelidad de los lectores más adictos. Ya no me creo sus informaciones", al tiempo que sostiene que El País "es un títere del Gobierno [...] sabemos que los diarios importantes están en manos del capital y del Gobierno. Es lógico que hagan lo que quieran con ellos, incluido echar e imponer directores" (El Plural, 2014).

La publicación Mongolia (2014) también es bastante afilada en sus comentarios sobre la sustitución de Moreno y afirma que "El País se ha ido tan a la derecha que cuesta imaginar cómo podría ser un nuevo giro, teniendo en cuenta que el 75\% del Consejo de Administración tiene vínculos directos con el sector financiero". Una opinión compartida por el periodista Enric Sopena.

El director de El Plural, en su artículo "'El País', de izquierda a derecha. Quo Vadis, Juan Luis?" señala que:

Todos los indicios apuntan a que el Gobierno de la derecha se ha aliado con Juan Luis Cebrián para salvar a El País. Pero el precio de este pacto es ominoso. El periódico de centro izquierda se ha convertido en un periódico de centro derecha. Pero con un añadido. El PP no es de centro derecha, sino de extrema derecha. (Sopena, 2014). 


\subsection{Repercusiones internacionales}

Las repercusiones en la prensa europea no han sido tan significativas como las generadas por la destitución de Pedro J. Ramírez y, prácticamente, se limitan a informar de forma aséptica sobre el cambio en la cúpula del diario. Sólo la prensa italiana destaca el giro a la derecha experimentado por el rotativo español y apunta a una "indulgencia con Rajoy" la destitución de Javier Moreno como director de El País.

Así, Europa Quotidiano, bajo el titular "El País svolta a destra. Il tramonto del 'giornale-partito"' (El País gira a la derecha. La puesta de sol del 'periódico de partido'), recoge que "El País ha vuelto a la tormenta. Mientras que el director va a cambiar, crece la controversia sobre el cambio en la política del periódico, acercándose cada vez más al gobierno de Rajoy" (Siniscalchi, 2014).

"Además de una disminución de la calidad periodística", en referencia a la publicación de las fotos falsas de Hugo Chávez en la cama del hospital, el rotativo prosigue:

La línea editorial ha supuesto un acercamiento a Rajoy [...] desde su elección en 2011, Cebrián no deja de hablar bien de Rajoy en cada oportunidad, además de mantener buenas relaciones con la vicepresidenta del Gobierno, Soraya Sáenz de Santamaría (Siniscalchi, 2014).

Incluso, señala que "promueve la idea de que el gobierno de España está dejando atrás la crisis" y que, desde la primera a la última página dedicada al Estado de la Nación, "estuvo acompañado por la foto de la sonrisa de Rajoy que eleva el pulgar hacia arriba en señal de victoria".

El artículo finaliza argumentando que "es de esperar que una cabecera histórica e importante como El País sea capaz de encontrar una manera de seguir existiendo a fin de garantizar la pluralidad de la información española, dominada por periódicos progubernamentales y de derecha" (Siniscalchi, 2014).

La posibilidad de que hubiesen más destituciones en la prensa española ya la apuntaba el Milano Finanza en su artículo "RSC, a rischio il direttore di El País", (RSC, un riesgo para el director de El País), en el que anunciaba que la sustitución de Pedro J. Ramírez podría causar un efecto dominó en el mercado editorial y que $E /$ País podría seguir "la revolución fijada por RCS para su principal diario español" (Montanari, 2014).

El diario italiano apuntaba a la posible sustitución de Moreno por Antonio Caño y que detrás estaría "la relación con la política y el gobierno de Rajoy, las mismas razones que en el mercado ibérico habrían provocado la expulsión de Ramírez de El Mundo" (Montanari, 2014).

En Francia, lo más significativo lo podíamos leer en Le Figaro (2014) donde Gauchet publicaba "Les mouvements dans les médias" (Los movimientos en los medios), un artículo aséptico en el que el rotativo hace un breve repaso al currículum de Antonio 
Caño, "el quinto director del periódico, fundado en 1976 por Juan Luis Cebrián, que iniciará sus funciones el próximo 4 de mayo, día del aniversario de la creación de El País".

En la prensa británica y alemana la repercusión ha sido nula, mientras que en el mercado anglosajón sólo la edición impresa del diario The New York Times recogía la noticia. El rotativo publicaba el 18 de febrero un artículo titulado "El País Outs Editor; 3rd Spanish Paper to Do So Amid Financial Woes" (El País expulsa a su editor; Tercer periódico en papel español que lo hace en medio de problemas financieros).

Firmado por Minder y Carvajal, señala la caída de la publicidad y de la venta de ejemplares como el motivo principal de la sustitución, sin embargo, no pierde oportunidad de mencionar que "los periódicos han continuado denunciando la corrupción sistémica y las cuentas bancarias en Suiza que han empañado a las principales instituciones de la sociedad española, incluyendo al gobierno, la monarquía y los titanes corporativos".

En este sentido, se hace eco de unas declaraciones de Pedro J. Ramírez en las que afirma "está claro que el gobierno y las poderosas instituciones económicas están aprovechando la crisis del modelo de negocio y la debilidad de los periódicos para tratar de controlar más y más información" (Minder y Carvajal, 2014).

Más repercusión encontramos en medios online Médium.com y Talking News Media. El primero de ellos, en su sección Media and the Press, recoge "Antonio Caño replaces Javier Moreno as Director of El País" (Antonio Caño sustituye a Javier Moreno como director de El País). La noticia indica que este cambio se produce tan solo dos semanas después de la destitución de Pedro J. Ramírez y que la llegada de Caño es vista como "un paso más en el cambio conservador del periódico" (Laurent, 2014).

Esta misma impresión es la que transmite Talking News Media quién, en un tono mucho más neutro, hace alusión a que la agitación dentro del panorama editorial español continúa con la sustitución de Moreno por el corresponsal en Washington del diario español. El rotativo apunta a que "la situación del negocio periodístico es complicada a causa de los problemas que todos conocemos" y afirma que "traer sangre nueva al trabajo no es una mala ided" (Hebbard, 2014).

Ahondando en materia, The World News (2014), en un artículo titulado "Antonio Caño sustituirá a Javier Moreno como director del diario "El País»" hace referencia a que la noticia se confirmó tras aparecer en los "dos últimos días en varios medios digitales, que apuntaron a un cambio de rumbo en la línea tradicionalmente progresista de uno de los rotativos más influyentes de España".

La información prosigue:

La noticia se conoce en España días después de la destitución de Pedro J. Ramírez, fundador de El Mundo. El ya exdirector del principal rival de El País apuntó entonces a la presión del gobierno conservador de Mariano Rajoy [...] en apenas dos semanas, los directores de dos de los diarios más importantes de España dejarán sus puestos (The World News, 2014). 
Debido a la enorme presencia que el grupo Prisa posee en Sudamérica, numerosos medios latinoamericanos recogen la sustitución de Moreno por Antonio Caño, considerado por muchos como el "virrey de América, por el poder que ostentaba al otro lado del Atlántico y la confianza que en él tenía depositada Juan Luis Cebrián, actuando a veces por encima del propio director del diario para el que trabajaba" (Libertad Digital, 2014).

Ello es debido, sin lugar a dudas a que Caño gestionaba personalmente importantes negocios de Prisa en Iberoámerica. No obstante, si a nivel nacional la prensa se muestra especialmente crítica con el relevo producido en la cúpula de El País, en Hispanoamérica los medios se hacen eco de esta sustitución de forma bastante impersonal.

Así, Caracol.com, de Venezuela, recoge que "el Consejo de Administración del diario español El País nombró hoy por unanimidad a Antonio Caño director del periódico en sustitución de Javier Moreno, informó el rotativo en una nota de prensa" (Caracol, 2014).

En la misma línea, El Nuevo Diario (2014) de Nicaragua titula: "El diario español El País con nuevo director". En la noticia desarrolla que "el Consejo de Administración del diario El País, nombró 'por unanimidad' este miércoles a Antonio Caño director del diario en sustitución de Javier Moreno".

Por su parte, Mundiario, El Primer Periódico Global de Análisis y Opinión (2014) destaca en su titular: "Antonio Caño será director de 'El País', a propuesta de Juan Luis Cebrián", Infobae América (2014): "Cambia la dirección del diario El país, el más leído de España" y Contactolatino.com (2014), "Antonio Caño, nombrado nuevo director de El País".

Sólo los diarios argentinos ahondan en las posibles causas que existen tras la destitución de Javier Moreno. Así, el diario Clarín publica que "la llegada al poder de Caño se interpreta como una continuidad al giro conservador sufrido por el periódico en los últimos años".

Esta significativa modificación en el periódico más importante de España llega poco tiempo después del cambio de mando en el diario El Mundo [...] los cambios recientes en El País también se interpretan como una continuidad en el giro conservador que evidenció el diario periódico en los últimos años. Desde la victoria del Partido Popular en las elecciones de 2011, el presidente del Grupo Prisa elogió la figura de Mariano Rajoy y sus relaciones con los medios de comunicación, en contraposición a la gestión de José María Aznar (Algañaraz, 2014).

También La Nación recoge que "El Mundo, segundo diario español en circulación, también cambió recientemente la conducción de su redacción" y apunta que la prensa española: 
Atraviesa una profunda crisis derivada de varios años de caída de los avisos publicitarios, en parte debido a la recesión en la mayoría de los países, y de la circulación de ejemplares impresos, afectada por las nuevas tecnologías y formas de consumo de noticias (La Nación, 2014).

\section{CONCLUSIONES}

La presente investigación pone de manifiesto cómo en el ámbito periodístico español el cambio de director de El País es percibido como consecuencia directa de influencias políticas y empresariales. Mientras que los medios impresos no se pronuncian al respecto, son los digitales, lo que dependen en menor medida de subvenciones y publicidad institucionales, los que apuntan a la crisis económica en la que está sumido el Grupo Prisa y a un cambio de tendencia ideológica en el rotativo como las causas ocultas tras la sustitución de Javier Moreno por Antonio Caño.

En el ámbito internacional, las repercusiones en la prensa alemana e inglesa es nula, mientras que algunos medios italianos y franceses apuntan a idénticos planteamientos que los medios online españoles y ven una maniobra política tras las sustituciones de los tres directores de los diarios impresos con más lectores en España: El País, El Mundo y La Vanguardia. Así, el Milano Finanza, por ejemplo, apunta a que la destitución viene dada por un acercamiento de posturas hacia el Gobierno de Rajoy, tal y como ocurrió con Pedro J. Ramírez.

Esta misma tendencia la encontramos en la prensa anglosajona y en los diarios argentinos La Nación y Clarín, mientras que el resto de medios latinoamericanos que recogen la noticia lo hacen desde un punto estrictamente informativo, llegando a reproducir fielmente el comunicado oficial de El País, algo que ocurre sobre todo en las cabeceras hispanoamericanas del Grupo Prisa, como es el caso de Caracol.

Del análisis realizado deducimos que el modelo mercantilista sigue funcionando en los medios impresos y que la prensa ya no es el cuarto poder, sino parte del primero al relacionarse directamente con cuestiones económicas y políticas.

\section{REFERENCIAS}

\subsection{LIBROS:}

Cachero, A. (2006). Elementos para una teoría comunicacional de la propaganda. Sevilla: Ediciones Alfar.

Castell, M. (2009). Comunicación y poder. Barcelona: Alianza.

De Fontcuberta, M. (1995). La noticia. Pistas para percibir el mundo. Barcelona: Paidós. 
Delgado, M. (1998). La globalización, ¿nuevo orden o crisis del viejo? Sevilla: Cuadernos de Economía y Sociedad.

Gomis, L. (1991). Teoría del periodismo. Cómo se forma el presente. Barcelona: Paidós Comunicación.

Labio, A. (2006). Comunicación, periodismo y control. Barcelona: Antrophos.

Marciano, M. (1992). Estructura y dinámica de la comunicación internacional. Barcelona: Bosch.

Quirós, F. (1991). Curso de estructura de la información. Madrid: Dossat.

\subsection{REVISTAS:}

Casero, A. (2009). El control político de la información periodística. Revista Latina de Comunicación Social, 64, pp. 354-366. Doi: 10.4185/RLCS-64-2009-828-354-366

Labio, A. (2001). La profesión periodística ante los intereses de la estructura informativa. Una aproximación al tema. Ámbitos, Revista Andaluza de Comunicación, 6, pp. 81-94.

Mosco, V. (1983). Entrevista con Herbert Schiller. Telos, 34, pp.117-135

Quirós, F. (1991). La estructura de la Información Periodística y sus fuentes bibliográficas documentales. Documentación de las Ciencias de la Información, 14, pp.307-312.

Reig, R. (2009). Bases teóricas y documentales para el estudio de la Estructura de la Información y el análisis estructural de los mensajes. Estudios sobre el Mensaje Periodístico, volumen 15, pp.385-407.

\subsection{REFERENCIAS WEBS:}

Algañaraz, J. C. (2014). Antonio Caño será el nuevo director del diario El País de España. Clarín. Recuperado de www.clarin.com/mundo/Antonio-Cano-director-PaisEspana 0 1087691263. Consultado el 18/02/2014.

Caracol. (2014). Antonio Caño es el nuevo director del diario español El País. Recuperado de www.caracol.com.co/noticias/entretenimiento/antonio-cano-es-elnuevo-director-del-diario-espanol-el-pais/20140226/nota/2101035. Consultado el 26/02/2014.

Contactolatino.com. (2014). Antonio Caño, nombrado nuevo director de El País. Recuperado de http://contacto-latino.com/redzin/2013-02/2014/02/26/antoniocano-nombrado-nuevo-director-de-el-pais-el-pais-com-espana. Consultado el 26/02/2014. 
Diario Crítico. (2014). Cebrián 'pliega' El País a Rajoy para salvar al Grupo Prisa. Recuperado de www.diariocritico.com/nacional/periodicos/cambios/450842. Consultado el 26/02/2014.

Ecoteuve. (2014). El 36\% de la plantilla de 'El País' rechaza a Antonio Caño como nuevo director. Recuperado de www.ecoteuve.eleconomista.es/ecoteuve/prensa/noticias/5569763/02/14/El-36-dela-plantilla-de-El-Pais-rechaza-a-Antonio-Cano-como-nuevo-director. Consultado el $14 / 02 / 2014$.

El Confindencial Digital. (2014). Un vídeo de la nueva subdirectora de El País alienta los temores: el periódico va a girar a la derecha. Recuperado de www.elconfidencialdigital.com/medios/subdirectora-Pais-temores-periodicoderecha. Consultado el 16/04/2014.

El Nuevo Diario. (2014). El diario español El País con nuevo director. Recuperado de www.elnuevodiario.com.ni/internacionales/312528-diario-espanol-pais-nuevodirector. Consultado el 26/02/2014.

El Plural. (2014). Maruja Torres tras el nombramiento de Caño: 'De 'El País' quedan restos y ruinas (...) Ya no me creo sus informaciones.' Recuperado de www.elplural.com/2014/03/15/maruja-torres-tras-el-nombramiento-de-cano-de-elpais\%C2\%B4-quedan-restos-y-ruinas-ya-no-me-creo-sus-informaciones. Consultado el $15 / 03 / 2014$.

Gauchet, Y. (2014). "Mouvements dans les medias". Le Figaro. http://www.lefigaro.fr/medias/2014/04/11/20004-20140411ARTFIG00229-lesmouvements-dans-les-medias.php 814/04)

Hebbard, D. B. (2014). El País names Antonio Caño its new editor. Talking New Media. Recuperado de www.talkingnewmedia.com/2014/02/19/el-pais-namesantonio-cano-its-new-editor. Consultado el 19/02/2014.

Infobae América. (2014). Cambia la dirección del diario El país, el más leído de España. Recuperado de www.infobae.com/2014/02/26/1546487-cambia-ladireccion-del-diario-el-pais-el-mas-leido-espana. Consultado el 26/02/2014.

Infoeuropa. (2014). La prensa en Europa. Informe. Recuperado de www.infoamerica.org/web1/informe/intro 4. Consultado el 14/05/2015.

Jordá, C. (2014). El País da la razón a la extrema derecha para desmarcarse de Zapatero. Recuperado de www.libertaddigital.com/nacional/2011-09-27/el-pais-dala-razon-a-la-derecha-extrema-para-desmarcarse-de-zapatero-1276436505/.

Consultado el 27/09/2011. 
La Mosca Mediática (2013). El País cambia de chaqueta ideológica y Lara desactiva a la extrema derecha mediática. Recuperado de www.lamoscamediatica.com/2013/05/el-pais-cambia-de-chaqueta-ideologicay.html. Consultado el 30/05/2014.

La Nación. (2014). Nuevo director de El País, de España. La Nación. Recuperado de www.lanacion.com.ar/1665311-nuevo-director-de-el-pais-de-espana. Consultado el 19/02/2014.

Lardiés, A. (2014). Cebrián ya tiene nuevo director para 'El País': Antonio Caño sustituirá a Javier Moreno. Recuperado de www.vozpopuli.com/economia-yfinanzas/38969-cebrian-ya-tiene-nuevo-director-para-el-pais-antonio-canosustituira-a-javier-moreno. Consultado el 16/02/2014.

Lardiés, A. (2014). La redacción de 'El País' castiga al nuevo director: Caño solo logra el $43 \%$ de los votos. Recuperado de www. vozpopuli.com/economia-yfinanzas/39413-la-redaccion-de-el-pais-castiga-al-nuevo-director-cano-solo-logra-el43-de-los-votos. Consultado el 14/02/2014.

Laurent, A. (2014). Antonio Caño replaces Javier Moreno as Director of El País". Medium. Recuperado de www.medium.com/@anto I/antonio-cano-replaces-javiermoreno-as-director-of-el-pais.. Consultado el 18/02/2014.

Libertad Digital. (2014). Cebrián destituirá al director de 'El Pais' en un nuevo acercamiento al gobierno. Recuperado de www.libertaddigital.com/espana/201402-16/cebrian-destituira-al-director-de-el-pais-en-un-nuevo-acercamiento-algobierno. Consultado el 18/02/2014.

Minder, R. y Carvajal, D. (2014). El País Outs Editor; 3rd Spanish Paper to Do So Amid Financial Woes. New York Times. Recuperado de www.nytimes.com/2014/02/19/world/europe/spanish-paper-el-pais-ousts-its-editor. Consultado el 18/02/2014.

Montanari, A. (2014). Rcs, a rischio il direttore di El Pais. Milano Finanza. Recuperado de www.milanofinanza.it/news/rcs-a-rischio-il-direttore-di-el-pais. Consultado el $17 / 02 / 2014$.

Mundidiario. (2014). Antonio Caño será director de 'El País', a propuesta de Juan Luis Cebrián. Recuperado de www.mundiario.com/articulo/sociedad/antonio-cano-seradirector-pais-propuesta-juan-luis-cebrian. Consultado el 19/02/2014.

Ortega, M. (2014). El País, cautivo y desarmado por Cebrián, se rinde a La Moncloa. Recuperado de www.periodistadigital.com/verdadmentira/2014/04/10/el-diario-elpais-cautivo-y-desarmado-por-cebrian-se-rinde-a-la-moncloa. Consultado el 08/04/2014. 
Periodista Digital (2014). La destitución de José Antich como director de La Vanguardia fue una imposición del Rey a Godó. Recuperado de www.periodistadigital.com/periodismo/prensa/2014/02/07/antich-godo-vanguardiarey-juan-carlos Consultado el 07/02/2014.

Periodista Digital. (2014). Sebastián ve la mano negra de Rajoy en el casi inminente cese de Javier Moreno en El País. Recuperado de www.periodistadigital.com/periodismo/prensa/2014/02/17/pablo-sebastian-el-paiscebrian-ussia-de-prada-isabel-san-sebastian-fumador. Consultado el 17/02/2014.

Prnoticias. (2014). La inversión en publicidad cae un 3,7\% en 2013: Prensa (13,5\%), Radio (-11\%), TV $(-6,2 \%)$. Recuperado de www.prnoticias.com/marketingpr/20128452-infoadex-2013-la-publicidad-enmedios-cae-un-8-prensa-135-radio-11-tv-62. Consultado el 18/05/2015.

Sanclemente, J. (2014). ¿Quién ha acabado con tres directores de periódico? Recuperado de www.eldiario.es/zonacritica/acabado-directores-periodico. Consultado el 18/02/2014.

Saénz, I. (2014). Por qué El País quiere irse más a la derecha. Recuperado de www.eldiario.es/rastreador/testamento-Pais-periodicos. Consultado el 01/03/2014.

Serrano, P. (2009). El Grupo Prisa se tambalea. Le Monde Diplomatique. Recuperado de www.atlanticaxxii.com/1269/la-crisis-de-el-pais-y-el-problema-democratico-delos-medios, Consultado el 01/03/2014.

Siniscalchi, E. (2014). El País svolta a destra. Il tramonto del "giornale-partito". Europa Quotidiano. Recuperado de www.europaquotidiano.it/2014/03/01/el-paissvolta-a-destra-la-fine-del-giornale-partito, Consultado el 01/03/2014.

Sopena, E. (2014). 'El País', de izquierda a derecha. Quo Vadis, Juan Luis?. Recuperado de www.elplural.com/opinion/el-pais-de-iquierda-a-derecha-quovadis-juan-luis. Consultado el 06/03/2014.

Soteras, J. (2014). El 'informe Caño' desata una crisis interna en el diario 'El País'. Recuperado de www.infolibre.es/noticias/medios/2014/02/16/el informe cano desata una crisis i nterna diario pais. Consultado el 16/02/2014.

The World News. (2014). Antonio Caño sustituirá a Javier Moreno como director del diario «El País». Recuperado de www.article.wn.com/view/2014/02/18/Antonio Cano sustituira a Javier Moreno c omo director del di. Consultado el 18/02/2014.

Toledo, D. (2014). 'El País' imita a 'El Mundo' en su volantazo pro-Gobierno Rajoy en pleno año electoral. Recuperado de 
www.elconfidencial.com/comunicacion/2014-02-17/el-pais-imita-a-el-mundo-en-suvolantazo-pro-gobierno-rajoy-en-pleno-ano-electoral. Consultado el 17/02/2014.

20 Minutos. (2012): Las claves de la crisis en 'El País' que ha abierto un cisma entre la cúpula directiva y la redacción. Recuperado de www.20minutos.es/noticia/1614319/0/claves/el-pais-prisa/ere-crisis. Consultado el $10 / 11 / 2012$.

Vozpópuli. (2014). La carta de Pedro J. que García-Abadillo no publica: "Rajoy es un crustáceo exánime. Recuperado de www.vozpopuli.com/economia-y-finanzas/52446-la-carta-de-pedro-j-que-garciaabadillo-no-publica-rajoy-es-un-crustaceo-exanime. Consultado el 09/11/2014.

Ymedia. (2015). Primer EGM 2015. Recuperado de http://www.ymedia.es/es/canalymedia/otros-medios/egm/953/abr-2015/1er-egm-2015. Consultado el 05/05/2015.

\section{AUTOR/ES:}

\section{Marián Alonso González.}

Marián Alonso González es Doctora en Comunicación por la Universidad de Sevilla (2008) con una Tesis Doctoral que versa sobre el cambio tecnológico de $A B C$ de Sevilla. Técnico de Comunicación en la Dirección de Comunicación y Gabinete de Presidencia de ADIF, es miembro del Grupo de Investigación Análisis y Técnica de la Información de la Universidad de Sevilla donde desarrolla su labor docente como profesora asociada de la Facultad de Comunicación impartiendo clases de Redacción Periodística Audiovisual. http://orcid.org/0000-0003-2676-0449 